\title{
The SARS-CoV-2 B.1.351 lineage (VOC $\beta$ ) is outgrowing the B.1.1.7 lineage (VOC $\alpha$ ) in some French regions in April 2021
}

Bénédicte Roquebert ${ }^{1}$, Sabine Trombert-Paolantoni ${ }^{1}$, Stéphanie Haim-Boukobza ${ }^{1}$, Emmanuel Lecorche ${ }^{1}$, Laura Verdurme ${ }^{1}$, Vincent Foulongne ${ }^{2}$, Mircea T. Sofonea3,4 , Samuel Alizon ${ }^{3,4}$

1. Laboratoire Cerba, Saint Ouen L'Aumône, France

2. Laboratoire de Virologie, CHU de Montpellier, Montpellier, France

3. MIVEGEC, CNRS, IRD, Université de Montpellier, Montpellier, France

4. These authors contributed equally to this article.

\section{Correspondence: Samuel Alizon (samuel.alizon@cnrs.fr)}

Citation style for this article:

Roquebert Bénédicte, Trombert-Paolantoni Sabine, Haim-Boukobza Stéphanie, Lecorche Emmanuel, Verdurme Laura, Foulongne Vincent, Sofonea Mircea T., Alizon Samuel. The SARS-CoV-2 B.1.351 lineage (VOC $\beta$ ) is outgrowing the B.1.1.7 lineage (VOC a) in some French regions in April 2021. Euro Surveill. T., Alizon Samuel. The SARS-CoV-2 B.1.351 lineage $($ VOC $\beta$ ) is outgrowing the B.1.1.7 line

To assess SARS-CoV-2 variants spread, we analysed 36,590 variant-specific reverse-transcription-PCR tests performed on samples from 12 April-7 May 2021 in France. In this period, contrarily to January-March 2021, variants of concern (VOC) $\beta$ (B.1.351 lineage) and/or $Y$ (P.1 lineage) had a significant transmission advantage over VOC a (B.1.1.7 lineage) in île-de-France (15.8\%; 95\% confidence interval (Cl):15.5-16.2) and Hauts-de-France $\quad(17.3 \% ; 95 \% \mathrm{Cl}: 15.9-18.7)$ regions. This is consistent with VOC $\beta$ 's immune evasion abilities and high proportions of prior-SARS-CoV-2-infected persons in these regions.

'Variants of concern' (VOC) are severe acute respiratory syndrome coronavirus 2 (SARS-CoV-2) phenotypically distinct lineages that are associated with major epidemiological or clinical shifts. To date, four have been classified as such by the World Health Organization (WHO) [1]. The first, $\mathrm{VOC}$ a, which corresponds to Pango lineage B.1.1.7, nextstrain clade 20l/501Y.V1, and GISAID clade/lineage GRY, is currently causing the majority of infections in Europe and North America [2], whereas, the second, VOC $\beta$ (Pango lineage: B.1.351; nextstrain clade: $20 \mathrm{H} / 501 Y . V 2$; GISAID clade/lineage: GH/501Y.V2) is the most common variant in South Africa [3]. The third variant, VOC Y (Pango lineage P.1; nextstrain clade: 20J/501Y.V3; GISAID clade/lineage: GR/501Y.V3) dominates in Brazil and South America [4] and the fourth VOC $\delta$ (Pango lineage: B.1.617.2; nextstrain clade: 21A/S:478K; GISAID clade/lineage: G/452R.V3) caused a major epidemic wave in India [5]. The outcome of the (indirect) competition between variants is yet open. In France, the early introduction of VOC $\beta$ in some regions makes it particularly important to monitor the spread of different variants [6].

\section{PCR testing of SARS-CoV-2-positive clinical samples for variants}

Since January 2021, the national guideline is to test all clinical samples that are positive for SARS-CoV-2 with an additional reverse-transcription (RT)-PCR to detect mutations indicative of certain variants $[7,8]$. Since April 2021, this variant-specific RT-PCR targets the N501Y mutation, which is shared by VOCs B.1.1.7, B.1.351 and P.1, and the $E 484 \mathrm{~K}$ mutation, which is found in VOCs B.1.351 and P.1, as well as the variant of interest (VOI) B.1.525 (WHO: $\mathrm{n}$; nextstrain clade: $20 \mathrm{~A} /$ S484K; GISAID clade/lineage: G/484K.V3) [1], but not in B.1.1.7.

We used the ID SARS-CoV-2/N501Y/E484K Quadruplex assay (ID Solution, Grabels, France) to test 53,687 SARS-CoV-2 positive samples collected between 12 April and 7 May 2021 in 13 French regions, with the majority of samples coming from the Île-deFrance region (Table 1). Some of the total samples (7-8\%, Table 1) originated from hospitals (mostly hospitalised patients), the rest from the general population. We only analysed data from individuals aged from 5 to 80 years to minimise in the analysis the weight of preschool children and elderly persons in long-term care facilities. $17.3 \%$ of the tests could not be interpreted; this was mainly because the cycle threshold (Ct) value was too high to ensure an equal sensitivity for the $\mathrm{N}_{501} \mathrm{Y}$ and $\mathrm{E}_{484} \mathrm{~K}$ targets. To avoid biasing the variant screening, all tests with $\mathrm{Ct}$ values strictly above 30 , including those where a lineage could be assigned, were ignored $(31.8 \% ; 17,097 / 53,687)$. Overall, we analysed $68.2 \%(36,590 / 53,687)$ of all the samples tested (Table 1). 
TABLE 1

Characteristics of the samples analysed via variant-specific reverse-transcription PCR, France, 12 April-7 May 2021 $(\mathrm{n}=36,590)$

\begin{tabular}{|c|c|c|}
\hline \multicolumn{2}{|l|}{ Characteristics of the samples } & Quantitative data \\
\hline \multicolumn{2}{|c|}{ Age in years of the persons providing a sample; number of samples $(95 \% \mathrm{Cl})$} & $38(10-73)$ \\
\hline \multirow{2}{*}{ Origin of the samples } & General population; number of samples (\%) & $34,022(93)$ \\
\hline & Hospital; number of samples (\%) & $2,568(7)$ \\
\hline \multirow{4}{*}{ Presence $(+)$ or absence $(-)$ of two mutations of interest } & 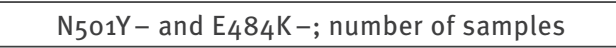 & 662 \\
\hline & 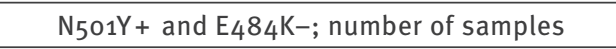 & 31,929 \\
\hline & 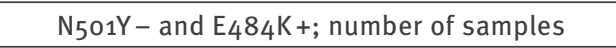 & 647 \\
\hline & 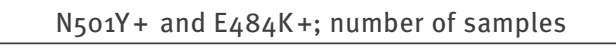 & 3,352 \\
\hline \multicolumn{2}{|l|}{ Ct of the real-time reverse-transcription PCR $(95 \% \mathrm{Cl})$} & $22.1(14.9-29.4)$ \\
\hline \multicolumn{2}{|l|}{ Sampling date $(95 \% \mathrm{Cl})$} & 21 Apr (12 Apr-6 May) \\
\hline \multirow{9}{*}{ Region } & Normandie; number of samples & 6,288 \\
\hline & Centre-Val de Loire; number of samples & 1,961 \\
\hline & Hauts-de-France; number of samples & 4,684 \\
\hline & Île-de-France; number of samples & 16,922 \\
\hline & Occitanie; number of samples & 409 \\
\hline & Provence-Alpes-Côte d'Azur; number of samples & 3,789 \\
\hline & Bourgogne-Franche-Comté; number of samples & 454 \\
\hline & Nouvelle-Aquitaine; number of samples & 1,444 \\
\hline & Other; number of samples & 639 \\
\hline
\end{tabular}

$\mathrm{Cl}$ : confidence interval.

\section{Sequencing profiles}

The specificity of the variant-specific RT-PCR we used is limited, since this PCR only targets two mutations. To gain additional insights regarding the type of variants circulating in the country, we sequenced $15 \%$ the samples collected on 30 March 2021 in France in this dataset for which the Ct was equal or lower than 28 using Twist Libraries and Illumina sequencing; the GISAID accession numbers are in Supplement $\mathrm{S}_{1}$. These samples were constituted for the most part (45\%; 215/478) by samples from the île-de-France region and showed a majority of viruses of the B.1.1.7 lineage (79.1\%, 378/478; Supplementary Table S1). The other prevalent lineages were B.1.351 (7.9\%, 38/478), B.1.525 $(4.4 \%, 21 / 478)$, and B.1.214 $(2.3 \%, 11 / 478)$, a lineage characterised by a variant not classified as a VOC, but which is under monitoring [9]. There were also lineages represented by less than $2 \%$ of the samples, such as P.1 $(0.6 \%, 3 / 478)$. In the subsets of samples from the Île-de-France $(n=215)$ and Hauts-de-France $(n=48)$ regions, the order of prevalence of the VOCs was the same as for the overall samples (Supplementary Table S1). Results from Santé Publique France, the French National Public Health Agency, for the Île-deFrance region in April ( $n=476$ samples) also generally agreed with these findings (Supplementary Table $\mathrm{S}_{1}$ ). Only a few samples from April were sequenced from the Hauts-de-France $(n=11)$ and île-de-France $(n=13)$ regions and for each of these regions, more than half of the samples were of the B.1.1.7 lineage.

Therefore, hereafter, samples with only the $\mathrm{N}_{501} \mathrm{Y}$ mutation detected are assumed to contain virus of B.1.1.7 lineage, samples with both $\mathrm{N}_{501} \mathrm{Y}$ and $\mathrm{E}_{484} \mathrm{~K}$ mutations, mainly virus of the B.1.351 lineage with possibly a minority of P.1, samples with only the E484K mutation, virus of the B.1.525 lineage, and samples with no mutation, wild type SARS-CoV-2 (although these samples may contain also viruses of B.1.214 lineage, which also lack the two mutations).

\section{Analysis of reverse-transcription PCR results}

Raw proportions of each SARS-CoV-2 lineage deduced by RT-PCR are shown in Figure 1 and raw numbers are shown in Supplementary Figure S1. As shown in both Figures, the sampling intensity in the dataset varies strongly across regions, which explains that some weeks have extreme values (e.g. in Occitanie). Overall, we see that lineage B.1.1.7 is dominant is most regions, and that the île-de-France is the region where the B.1.351 and/or P.1 lineages are the most frequently detected.

\section{Ethical statement}

This study has been approved by the Institutional Review Board of the CHU of Montpellier and is registered at ClinicalTrials.gov with identifier NCTo4738331.

\section{Lineage spreading in France}

We used a multinomial log-linear model (the multinom function from the nnet R package [10]) to identify factors associated with the detection of certain lineages (B.1.1.7 being the variant of reference). The explanatory variables were the individual's age (type of variable: integer), origin of the sample (hospital or community; 


\section{FIGURE 1}

Raw daily cumulative frequencies of variant-specific reverse-transcription PCR test results for SARS-CoV-2 in eight French regions, 12 April-7 May $2021(\mathrm{n}=33,583)^{\mathrm{a}}$

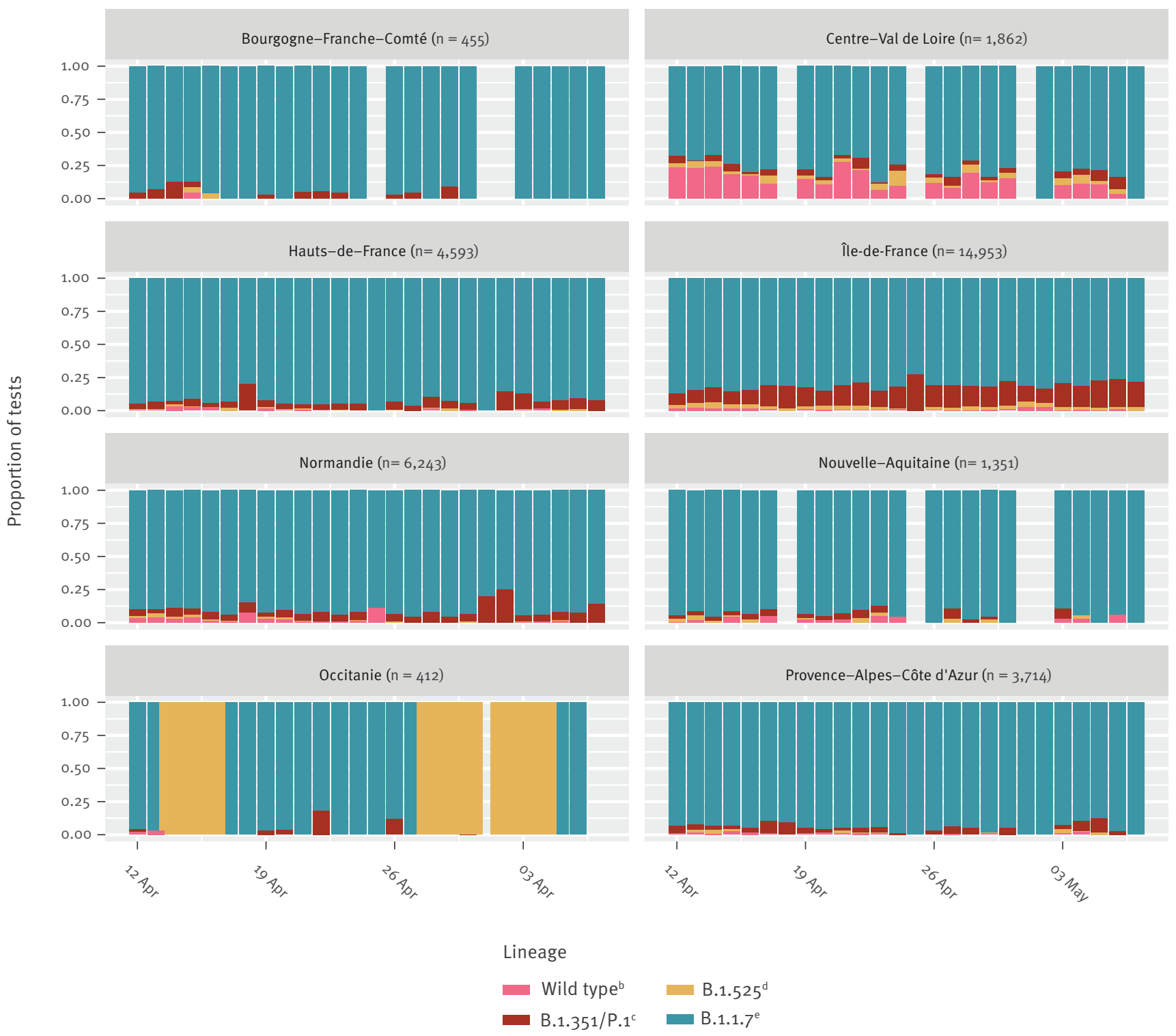

SARS-CoV-2: severe acute respiratory syndrome coronavirus 2; VOC: variant of concern; VOI: variant of interest.

a Only regions with more than 400 respective tests are shown.

${ }^{b}$ Characterisation as wild type SARS-CoV-2 is based on the absence of both $\mathrm{N}_{501} \mathrm{Y}$ and E484K mutations.

${ }^{c}$ Characterisation as B.1.351 and/or P.1 lineage (VOC $\beta$ and/or $\gamma$ ) is based on the presence of both N501Y and E484K mutations.

${ }^{d}$ Characterisation as B.1.525 lineage (VOI n) is based on the simultaneous absence of $\mathrm{N}_{501} \mathrm{Y}$ and presence of E484K mutation.

${ }^{e}$ Characterisation as B.1.1.7 lineage ( $\mathrm{VOC}$ a) is based on the simultaneous presence of $\mathrm{N}_{501} \mathrm{Y}$ and absence of $\mathrm{E}_{4} 84 \mathrm{~K}$ mutation.

The number of tests performed is indicated in each panel. For each day, the different colours indicate the proportion of tests belonging to each of the four screening categories (these sum to 1.0 every day). Regions with few tests can exhibit strong variations in frequencies for some days (e.g. Occitanie for days with only B.1.525 detected). See Supplementary Figure S1 for the raw numbers instead of the proportions. 
Factors associated with the detection of certain SARS-CoV-2 lineages, as assessed by relative risk ratios using a multinomial log-linear model, France, 12 April-7 May $2021(\mathrm{n}=36,590)$

\begin{tabular}{|c|c|c|c|c|}
\hline \multirow{4}{*}{ Factor } & & \multicolumn{3}{|c|}{ Median RRR with significance $(95 \% \mathrm{Cl})$ for the RRR } \\
\hline & & Wild type & B.1.351/P1 & B.1.525 \\
\hline & & & $(\operatorname{VOC} \beta / \gamma)$ & $(\mathrm{VOI} n)$ \\
\hline & & $\mathrm{N}_{501} \mathrm{Y}-/ \mathrm{E}_{484 \mathrm{~K}-}$ & $\mathrm{N}_{501} \mathrm{Y}_{+} / \mathrm{E}_{484} \mathrm{~K}+$ & $\mathrm{N}_{501} \mathrm{Y}-/ \mathrm{E}_{484} \mathrm{~K}+$ \\
\hline \multicolumn{2}{|l|}{ Age (increase per year) } & NS (0.88-1.00) & $0.95^{\mathrm{b}}(0.92-0.98)$ & $0.86^{\mathrm{b}}(0.80-0.93)$ \\
\hline \multirow{2}{*}{ Origin of the samples } & Non-hospital & Reference & Reference & Reference \\
\hline & Hospital & NS $(0.67-1.30)$ & $1.56^{\mathrm{a}}(1.40-1.80)$ & NS (0.64-1.30) \\
\hline \multirow{9}{*}{$\begin{array}{l}\text { Interaction between sampling } \\
\text { region and calendar date of } \\
\text { sampling }\end{array}$} & Normandie & $0.54^{a}(0.43-0.63)$ & NS (0.95-1.10) & $0.69^{\mathrm{a}}(0.55-0.81)$ \\
\hline & Centre-Val de Loire & $0.26^{\mathrm{a}}(0.18-0.30)$ & NS (0.84-1.10) & NS (0.90-1.70) \\
\hline & Hauts-de-France & $0.72^{\mathrm{b}}(0.57-0.91)$ & $1.14^{\mathrm{c}}(1.00-1.30)$ & NS $(0.74-1.20)$ \\
\hline & île-de-France & $0.74^{\mathrm{a}}(0.64-0.82)$ & $1.42^{\mathrm{a}}(1.40-1.50)$ & $0.81^{a}(0.71-0.91)$ \\
\hline & Nouvelle-Aquitaine & NS $(0.74-1.60)$ & $1.25^{\mathrm{c}}(1.10-1.50)$ & NS (0.57-1.20) \\
\hline & Occitanie & NS $(0.35-1.40)$ & NS $(0.76-1.40)$ & NS $(0.60-2.30)$ \\
\hline & Provence-Alpes-Côte d'Azur & NS (o.67-1.10) & NS $(0.95-1.20)$ & NS $(0.74-1.20)$ \\
\hline & Bourgogne-Franche-Comté & NS $(0.38-1.70)$ & NS (0.55-1.10) & NS $(0.34-1.50)$ \\
\hline & Other & NS (0.50-1.70) & NS $(0.80-1.40)$ & NS $(0.42-1.40)$ \\
\hline
\end{tabular}

$\mathrm{Cl}$ : confidence interval, NS: non-significant; VOC: variant of concern; VOI: variant of interest, RRR: relative risk ratios; SARS-CoV-2: severe acute respiratory syndrome coronavirus 2 .

The minus (-) sign after a mutation indicates its absence; the plus (+) its presence.

The reference lineage in the analysis is B.1.1.7 ( $\left.\mathrm{N}_{501} \mathrm{Y}+/ \mathrm{E}_{484} \mathrm{~K}-\right)$, i.e. the VOC a.

a $p<0.001$.

${ }^{\mathrm{b}} \mathrm{p}<0.01$.

${ }^{c} p<0.05$.

$P$ values are obtained using a two-tailed z-test.

type of variable: binary), and the interaction between the sampling region (type of variable: categorical) and the calendar date of sampling (treated as an integer) (Table 2). Integer values were centred and scaled. Details about the statistical methods are presented in Supplement S2. The raw data and R script used are also provided as Supplementary data.

The multinomial model revealed differences between lineages (Table 2). In terms of age, we found that older patients had a lower risk of being infected by B.1.351/P.1 and B.1.525 than by B.1.1.7 (our reference). In hospital settings, we found an over-representation of B.1.351/P.1 compared with B.1.1.7. When analysing region-specific temporal trends, we found that, for all regions, the risks of being infected by a wild type or a B.1.525 virus were either identical or lower than the risk of being infected by B.1.1.7. Conversely, we found that the risk of being infected by B.1.351/P.1 instead of B.1.1.7 significantly increased with time in île-deFrance, and to a lesser extent in Hauts-de-France and Nouvelle-Aquitaine.

\section{Transmission advantage of B.1.351/P.1 vs}

\section{B.1.1.7}

Trends from the multinomial model should be treated with caution because of autocorrelation issues. Therefore, to investigate the temporal trends, we used the method described in [11] and, for each region of interest, fitted a logistic growth model to the fitted values of a generalised linear model (GLM) with three factors on the data sampled outside hospitals. In addition to the sampling date and the individual age, we also added the department (i.e. a within-region administrative unit), where the sample was performed. For simplicity, we tested the transmission advantage of B.1.351/P.1 compared with B.1.1.7 and neglected the other lineages in the analysis. We performed the analysis only in the île-de-France, Hauts-de-France, and Nouvelle-Aquitaine regions. With few data regarding the coronavirus disease (COVID-19) epidemic serial interval in France, i.e. the time between the onset of the symptoms in an individual and that in a person he/ she infects, we used the one from [12].

We found a transmission advantage of $15.8 \%$ (95\% confidence interval: $15.5-16.2 \%$ ) in Île-de-France and 17.3\% (95\% Cl: 15.9-18.7\%) in Hauts-de-France (Figure 2). In Nouvelle-Aquitaine, the logistic growth model was not significant, which could be due to the fact that this region was less affected by the third epidemic wave than the other two [13].

\section{Discussion}

When analysing the results of variant-specific tests on samples obtained from January to March 2021, we found that the B.1.1.7's (VOC a) transmission advantage relative to wild type lineages was larger than 


\section{FIGURE 2}

Estimated evolution of proportions of B.1.351/P.1 ${ }^{\text {a }}$ (VOC $\beta / \gamma$ ) with respect to B.1.1.7 (VOC $\alpha$ ), excluding other lineages, in Île-de-France and Hauts-de-France, France, 9 April-28 May 2021

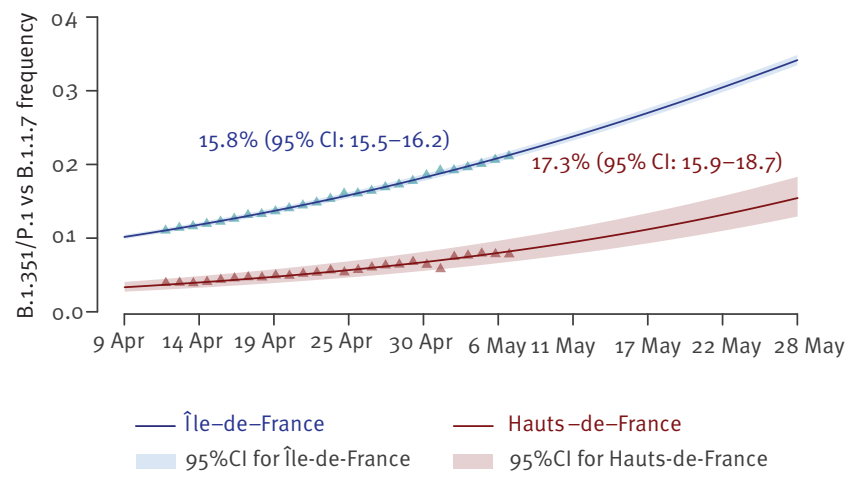

$\mathrm{Cl}$ : confidence interval; GLM: generalised linear model; VOC: variant of concern.

a The reverse-transcription PCR used to detect the different variants cannot distinguish between P.1 (VOC Y) and B.1.351 (VOC $\beta$ ) lineages; based on the sequencing of a subset of samples in March and April however, the B.1.351 (VOC $\beta$ ) appears to dominate over the P.1 (VOC $\gamma$ ) lineage in France and in the regions considered. Therefore the results are considered as likely reflecting mostly the B.1.351 variant (VOC $\beta$ ) spread.

Each region is represented in a different colour, with the triangles indicating the GLM-fitted values and the line the output of the logistic growth model estimation. The shaded area in the same colour as the line represents the $95 \% \mathrm{Cl}$. The text indicates the estimated transmission advantage of B.1.351 (VOC $\beta$ ) with respect to $\mathrm{B} \cdot 1.1 .7$ (VOC a) and the $95 \% \mathrm{Cls}$.

that of $\mathrm{B} .1 .351$ (VOC $\beta$ ) relative to wild type lineages [14]. During April 2021, in at least two French regions, this trend appears to have shifted with B.1.351 (VOC $\beta$ ) and possibly P.1 (VOC $\gamma$ ) spreading more rapidly than B.1.1.7. The B.1.351 lineage has known immune evasion properties $[15,16]$. Therefore, île-de-France being one of the French regions the most impacted to date by the epidemic [13], it is possible that a shift in variants with a transmission advantage is occurring there, because of the high proportion of individuals with immunity acquired through prior-SARS-CoV-2-infections. Vaccination might favour immune escape mutants [17] but the coverage with COVID-19 vaccines is homogeneous among French regions. Our results call for more detailed analyses regarding the link between the transmission advantage of the B.1.351 variant and the proportion of the population with immunity (following infection or vaccination) in different French regions.

There are some limitations to this analysis. First, although we performed sequencing to distinguish between B.1.351 and P.1 lineages, in some samples collected in March and, to a lesser extent, April, further sequencing will be needed to validate our assumption that the transmission advantage belongs to B.1.351, to P.1, or to both. Second, France had entered a third national lockdown on 3 April, which means that most of the tests analysed here were performed in a declining epidemic [18]. If what we assume to be the B.1.351 lineage causes infections that have a shorter generation interval than the B.1.1.7 lineage, this could affect the transmission advantage estimates. While there are some data on generation intervals for COVID-19 epidemics in France [19], studies are so far limited. Furthermore, analyses performed on the detailed United Kingdom epidemic data found that the hypothesis of differences in generation interval between B.1.1.7 and wild type lineages was less likely than other hypotheses, especially differences in contagiousness [2]. Finally, it is unlikely that non-pharmaceutical interventions would affect differently the transmission of the variants.

In conclusion, given the progressive lifting of the control measures in June 2021 in France [18], these results call for particular care regarding vaccination rollout and the maintenance of non-pharmaceutical prevention until vaccine coverage reaches levels compatible with spontaneous regression of the epidemic.

\section{Acknowledgements}

We thank the ETE modelling team for discussion, as well as the CNRS, the IRD, the ANR, and the Région Occitanie for funding (PHYEPI grant). We thank the Infectious diseases department in Cerba laboratory, and the technical and IT teams for their technical assistance.

Funding statement: We thank the CNRS, the IRD, the ANR and the Région Occitanie (PHYEPI project) for funding.

\section{Conflict of interest}

None declared.

\section{Authors' Contributions}

Bénédicte Roquebert, Sabine Trombert-Paolantoni, Stéphanie Haim-Boukobza, Emmanuel Lecorche, Vincent Foulongne, and Laura Verdurme collected the RT-PCR data. Mircea T. Sofonea and Samuel Alizon performed the statistical analysis. Samuel Alizon wrote the first version of the article. All the authors contributed to the final version of the manuscript.

\section{References}

1. World Health Organization (WHO). Tracking-SARS-CoV2-variants. Geneva: WHO; 2021. [Accessed 1 Jun 2021]. Available from: https://www.who.int/en/activities/ tracking-SARS-CoV-2-variants/

2. Davies NG, Abbott S, Barnard RC, Jarvis Cl, Kucharski AJ, Munday JD, et al. , CMMID COVID-19 Working Group, COVID-19 Genomics UK (COG-UK) Consortium. Estimated transmissibility and impact of SARS-CoV-2 lineage B.1.1.7 in England. Science. 2021;372(6538):eabg3055. https://doi.org/10.1126/science. abg3055 PMID: 33658326

3. Tegally H, Wilkinson E, Giovanetti M, Iranzadeh A, Fonseca V, Giandhari J, et al. Emergence and rapid spread of a new severe acute respiratory syndrome-related coronavirus 2 (SARSCoV-2) lineage with multiple spike mutations in South Africa. medRxiv. 2020;2020.12.21.20248640. Faria NR, Mellan TA, Whittaker C, Claro IM, Candido DDS, Mishra S, et al. Genomics and epidemiology of the P.1 SARS-CoV-2 lineage in Manaus, 
Brazil. Science. 2021;372(6544):815-21.https://doi.org/10.1126/ science.abh2644 PMID: 33853970

4. Faria NR, Mellan TA, Whittaker C, Claro IM, Candido DDS, Mishra S, et al. Genomics and epidemiology of the P.1 SARSCoV-2 lineage in Manaus, Brazil. Science. 2021;372(6544):81521. https://doi.org/10.1126/science.abh2644 PMID: 33853970

5. GISAID. "Tracking of Variants". gisaid.org. GISAID. [Accessed 30 May 2021]. Available from: https://www.gisaid.org/ hcov19-variants/

6. SARS-CoV-2 variant with lineage B.1.351 clusters investigation team. Linked transmission chains of imported SARS-CoV-2 variant B.1.351 across mainland France, January 2021. Euro Surveill. 2021;26(13):2100333. PMID: 33797392

7. Haim-Boukobza S, Roquebert B, Trombert-Paolantoni S, Lecorche E, Verdurme L, Foulongne V, et al. Detecting Rapid Spread of SARS-CoV-2 Variants, France, January 26-February 16, 2021. Emerg Infect Dis. 2021;27(5):1496-9. https://doi. org/10.3201/eid2705.210397 PMID: 33769253

8. Gaymard A, Bosetti P, Feri A, Destras G, Enouf V, Andronico A, et al. Early assessment of diffusion and possible expansion of SARS-CoV-2 Lineage 20l/501Y.V1 (B.1.1.7, variant of concern 202012/01) in France, January to March 2021. Euro Surveill. 2021;26(9):2100133. https://doi.org/10.2807/1560-7917. ES.2021.26.9.2100133 PMID: 33663644

9. European Centre for Disease Prevention and Control (ECDC). SARS-CoV-2 variants of concern as of 24 May 2021. Stockholm: ECDC; 2021. Available from: https://www.ecdc.europa.eu/en/ covid-19/variants-concern

10. R Core Team. R: A Language and Environment for Statistical Computing. Vienna: R Foundation for Statistical Computing; 2015. Version 4.0.5. Available from: http://www.R-project.org/

11. Chevin LM. On measuring selection in experimental evolution. Biol Lett. 2011;7(2):210-3. https://doi.org/10.1098/ rsbl.2010.0580 PMID: 20810425

12. Nishiura $H$, Linton NM, Akhmetzhanov AR. Serial interval of novel coronavirus (COVID-19) infections. Int J Infect Dis. 2020;93:284-6. https://doi.org/10.1016/j.ijid.2020.02.060 PMID: 32145466

13. Le Vu S, Jones G, Anna F, Rose T, Richard J-B, Bernard-Stoecklin $\mathrm{S}$, et al. Prevalence of SARS-CoV-2 antibodies in France: results from nationwide serological surveillance. Nat Commun. 2021;12(1):3025. https://doi.org/10.1038/s41467-021-23233-6 PMID: 34021152

14. Roquebert B, Haim-Boukobza S, Trombert-Paolantoni S, Lecorche E, Verdurme L, Foulongne V, et al. SARS-CoV-2 variants of concern are associated with lower RT-PCR amplification cycles between January and March 2021 in France. medRxiv. 2021;2021.03.19.21253971.

15. Zhou D, Dejnirattisai W, Supasa P, Liu C, Mentzer AJ, Ginn HM, et al. Evidence of escape of SARS-CoV-2 variant B.1.351 from natural and vaccine-induced sera. Cell. 2021;184(9):23482361.e6. https://doi.org/10.1016/j.cell.2021.02.037 PMID: 33730597

16. Li Q, Nie J, Wu J, Zhang L, Ding R, Wang H, et al. SARSCoV-2 501Y.V2 variants lack higher infectivity but do have immune escape. Cell. 2021;184(9):2362-2371.e9. https://doi. org/10.1016/j.cell.2021.02.042 PMID: 33735608

17. Hacisuleyman E, Hale C, Saito Y, Blachere NE, Bergh M, Conlon EG, et al. Vaccine Breakthrough Infections with SARS-CoV-2 Variants. N Engl J Med. 2021;NEJMoa2105000. https://doi. org/10.1056/NEJMoa2105000 PMID: 33882219

18. Sofonea MT, Boennec C, Michalakis Y, Alizon S. Two waves and a high tide: the COVID-19 epidemic in France. Anaesth Crit Care Pain Med. 2021;40(3):100881. https://doi.org/10.1016/j. accpm.2021.100881 PMID: 33962051

19. Paireau J, Mailles A, Eisenauher C, de Laval F, Delon F, Bosetti P, et al. Early chains of transmission of COVID-19 in France. MedRxiv. 2020;2020.11.17.20232264.

\section{License, supplementary material and copyright}

This is an open-access article distributed under the terms of the Creative Commons Attribution (CC BY 4.0) Licence. You may share and adapt the material, but must give appropriate credit to the source, provide a link to the licence and indicate if changes were made.

Any supplementary material referenced in the article can be found in the online version.
This article is copyright of the authors or their affiliated institutions, 2021. 